\title{
Rosie Findley (éd.), The Work Ethic and the Scots / L'Éthique du travail et les Écossais, dans Civilisations, $\mathrm{n}^{\mathrm{o}} 12$
}

Presses de l'université de Toulouse 1 Capitole, 2013, 173 p.

\section{Pierre Morère}

\section{(2) OpenEdition \\ Journals}

Édition électronique

URL : http://journals.openedition.org/etudesecossaises/1031

DOI : 10.4000/etudesecossaises. 1031

ISSN : 1969-6337

\section{Éditeur}

UGA Éditions/Université Grenoble Alpes

\section{Édition imprimée}

Date de publication : 25 avril 2015

Pagination : 186-190

ISBN : 978-2-84310-296-7

ISSN : $1240-1439$

\section{Référence électronique}

Pierre Morère, «Rosie Findley (éd.), The Work Ethic and the Scots / L'Éthique du travail et les Écossais, dans Civilisations, n० 12 », Études écossaises [En ligne], 17 | 2015, mis en ligne le 25 avril 2016, consulté le 17 mars 2021. URL : http://journals.openedition.org/etudesecossaises/1031 ; DOI : https://doi.org/ 10.4000/etudesecossaises.1031 
le Supplement de 1825. Rennie fait également état d'additifs de Jamieson jamais publiés, mais qui demeurent très instructifs pour le chercheur.

Quant à la postérité du dictionnaire, Rennie cite indirectement les propos de Willa Muir lors du centenaire de la mort de Jamieson :

The power of the Scots language recorded in the Dictionary was timeless, and far outweighed the fact that, in other respects, the dictionary was now out of date. The Dictionary, she affirms, was a "valient barrier" which Jamieson had raised against the "gradual draining away of old Scotland". (p. 236)

Le grand mérite de ce livre de Susan Rennie, c'est d'abord qu'il renferme un trésor de renseignements sur la façon de présenter la langue écossaise à partir de l'origine des mots jusqu'à leur usage contemporain. Cet ouvrage s'adresse certes en priorité aux philologues et aux linguistes, mais l'auteur a su éviter l'écueil d'une trop grande technicité et son propos est parfaitement abordable par le non-spécialiste. La seule réserve que l'on pourrait avancer, c'est la prudence ou ce que l'on pourrait appeler la «neutralité scientifique» dont Susan Rennie fait preuve. En effet, les choix de Jamieson, et en particulier celui de bien distinguer les origines des langues anglaise et écossaise, peuvent certes se fonder sur le résultat de ses recherches, mais il est difficile aussi de ne pas supposer qu'ils aient été inspirés par des motivations d'ordre idéologique favorable à la conception d'une culture distincte de l'Écosse.

\section{Pierre Morère}

Univ. Grenoble Alpes

Rosie Findley (éd.), The Work Ethic and the Scots / L'Éthique du travail et les Écossais, dans Civilisations, n ${ }^{\circ}$ 12, Presses de l'université de Toulouse 1 Capitole, 2013, 173 pages, 15 euros.

Le thème de l'éthique du travail choisi pour le dixième congrès de la Société française d'études écossaises à Toulouse en 2010, et dont ce recueil publie les communications qui y furent présentées, offre un intérêt majeur. D'abord parce qu'il traite, de façon diachronique, l'impact d'une morale issue du calvinisme, et ensuite ses divers avatars et mutations au fil du temps dans toute leur complexité. L'idée centrale qui se retrouve dans les 
articles de ce recueil, est que l'éthique du travail s'alimente à des sources à la fois spirituelles et religieuses.

Dans la préface, Rosie Findlay allie joliment, dans sa présentation des dix articles composant le recueil selon un ordre chronologique, le sérieux qui s'impose et une note d'humour particulièrement bienvenue à propos d'un sujet qui s'annonce austère : «Yet, it also has to be recognised, the Scots have distinguished themselves by another attitude to work which seems to counterbalance the excesses of the Calvinist mind set and mark them out as the great champions of the "good life" or nobody's fools. » (p. 10)

Le premier article de Christian Jérémie (université de Saint-Étienne) ouvre le débat dans le domaine de l'histoire des idées : «John Knox : ce qu'est un homme et son œuvre selon Thomas Carlyle». L'intérêt de cette étude est de traquer l'influence d'un calvinisme toujours présent, mais apparemment relégué dans l'ombre, chez l'auteur du Sartor Resartus (1836) et qui transparaît dans son étude du chartisme. L'auteur écrit : «Carlyle assimile le travail à une forme de sacerdoce qu'on a envie de qualifier d'universel, chaque travailleur étant à la fois prêtre et roi. L'idée que prêtrise et royauté, elles-mêmes combinées, comme on sait, dans la personne du Christ, soient confiées à la responsabilité de chaque homme dans le travail, peut amener à déceler l'écho d'une christologie latente chez Carlyle, ou du moins la trace d'une éducation chrétienne.» (p. 24)

Le second article de Laura Hedrick (University of St Andrews) reste dans le domaine religieux avec «Presbyterian Work Ethic and the Calvinists to the North: Scottish Reality or European Stereotype?». L'auteur évoque la réputation austère d'âpreté au travail des Écossais particulièrement visible dans les domaines du commerce et des armées et se demande si elle découle de leur volonté de s'afficher comme tels où si elle est le résultat d'un stéréotype. Elle conclut : «Despite its occasional usefulness, however, Scottish Presbyterian work ethic is likely a national myth developed from international perception of Scottish behaviour», et ce mythe persiste au point d'intéresser toujours les chercheurs.

Clarisse Godard Desmarest (université de Picardie) se penche sur les rapports entre l'esthétique et l'utilitaire dans l'architecture et les paysages dans son article «The Work Ethic in the Improvement of Scottish Houses and Landscapes of the 18th Century». Elle évoque l'influence du style palladien qui rencontre un écho favorable en Écosse dès lors qu'il unit harmonieusement l'utile et l'agréable, contrairement à ce qui se passe dans l'Angleterre du XVIII ${ }^{\mathrm{e}}$ siècle. L'auteur cite notamment le cas de John Erskine (1675-1732) dont le projet entendait allier dans le paysage industrie et beauté à Alloa (p. 49). Elle explique avec raison que Erskine partageait les idées esthétiques de David Hume et d'Adam Smith, mais elle 
aurait pu ajouter que la notion d'improvement qu'elle étudie était aussi particulièrement chère à Shaftesbury.

Christian Auer (université de Strasbourg) aborde le XIX ${ }^{\mathrm{e}}$ siècle avec le sujet «L'aide apportée aux victimes de la famine dans les Hautes Terres d'Écosse entre 1847 et 1850 ou le travail comme vecteur de contrôle social». Il explique l'objectif double du Central Board of Management of the Fund for the Relief the Destitute Inhabitants of the Highlands qui consiste autant à venir en aide aux déshérités qu'à les conduire à adhérer à un système de valeurs permettant de mieux les contrôler. Tout en reconnaissant que l'institution du Central Board répondait bien au départ à des impératifs humanitaires, l'auteur précise aussi que les intentions furent par la suite moins louables : «Mais il serait impossible d'occulter le fait que cette intervention se situa dans le cadre d'un objectif plus large consistant à modifier et à transformer de façon pérenne le mode de vie des individus auxquels on venait en aide, d'où la nécessité de recourir à des stratégies de contrôle, ce qui bien évidemment modifie radicalement la perspective que l'on peut avoir sur le processus. » (p. 65)

Tri Tran (université de Tours) analyse un aspect un peu négligé des relations entre patronat et ouvriers dans les chantiers navals de la Glyde au cours du «long» XIX siècle dans son article «The Work Ethic at the John Brown Shipbuilding and Engeneering Company: 1847-1918». L'auteur rappelle que la recherche couvrant la même période s'est surtout intéressée aux luttes ouvrières opposant travailleurs non spécialisés et patrons, et il s'interroge sur les raisons pour lesquelles dans les chantiers navals les grèves ne furent guère fréquentes. Il conclut que cette forme de consensus repose, non seulement sur des conditions de travail plutôt convenables, mais aussi et surtout sur un partage de valeurs communes entre patrons et ouvriers. Tri Tran précise : «The vast majority of members of the Church of Scotland and of the dissenting churches were mainly skilled working class: a religious census carried out in 1891 showed that 4,745 inhabitants of Clydebank (or 70\% of the population) were members of local churches, and those attending at least once on a Sunday numbered 3,760 (or 56\%).» (p. 74)

Jean Berton (université Toulouse 2) étudie les rapports entre éthique et travail à travers une œuvre littéraire majeure dans son article «L'éthique du travail chez les Gaëls d'Écosse dans les textes de Iain Crichton Smith». Tout en rappelant que ce poète n'a pas toujours été des plus tendres dans sa critique des Gaëls écossais, J. Berton montre que le thème de la finalité du travail, inspiré par la pensée existentialiste, est constant chez Crichton Smith depuis le roman des débuts Consider the Lilies jusqu'à sa dernière œuvre théâtrale Lazybed et, à partir de ce constat, il montre les différences entre gaélophones et scotto/anglophones. Pour illustrer son propos, l'au- 
teur de l'article analyse le personnage de Meurdoch MacRae dans le récit romancé In the Middle of the Wood (1987) qui se flatte de ne pas aimer le travail et qui, partant, s'oppose au système de valeurs dominant. En réponse à la réputation de fainéantise des Gaëls, J. Berton explique que «Crichton Smith remet en cause l'éthique du travail rémunérateur imposée par une culture étrangère» (p. 90).

C'est encore à Crichton Smith que Stéphanie Noirard consacre son article sur le sujet : "Gutting Herring in My Poetry": The Poet(h)ics of Iain Crichton Smith Assimilating and Confronting a Calvinistic Work Ethic». L'auteur étudie l'influence d'une éducation calviniste dans l'île de Lewis sur la création poétique, et comment peuvent se concilier, dans une éthique du travail, l'art et le travail manuel. S. Noirard explique comment le poète est perçu d'emblée comme un être oisif qui n'accepte pas, comme la Bible le lui enjoint, de gagner sa vie «à la sueur de son front». La thèse très originale que soutient l'auteur est que Crichton Smith, tout pourfendeur du calvinisme qu'il soit, n'en subit pas moins fortement son influence : «The transgression, or rather the step beyond the Puritan ethic of Lewis however, shows Crichton Smith's writing even more influenced by Protestantism than he thought, but also perhaps more posetively so. Though he certainly was unaware of it, his own poetic reformation is close to a Calvinian ethic. » (p. 107)

L'article suivant (dont l'abstract ne figure d'ailleurs pas en fin d'ouvrage), rédigé par Ian Brown (Kingston University, Londres), a pour titre : "Nationhood and Affective Communities in Late Twentieth-Century Scottish Workplace Drama». Le rapport éthique/travail est ici étudié dans sa représentation au théâtre après 1970. L'auteur se penche sur cinq pièces: Willie Rough, The Bevellers, The Slab Boys, Bondagers et The Cut. I. Brown conclut son analyse de ces œuvres théâtrales en soulignant l'ambiguïté du thème de la vertu du travail : «[...] we recognise through them that simple ideas about the virtue of the work ethic or workplace loyalty are evasions of the true complexities and ambiguities of economics and society that underlie work. » (p. 137)

C'est dans une optique très contemporaine que David Clark (université de La Corogne, Espagne) étudie comment les questions de genre et de religion influent sur la vie des personnages féminins dans les romans de Denise Mina, dans l'article «Leaving Wasn't Always an Option: Work, Religion and Gender in the Novels of Denise Mina». Ces romans dont l'action se situe dans le milieu irlandais de Glasgow se concentrent sur la condition des ouvrières confrontées à la double pression exercée par le monde masculin et le contexte protestant. L'intérêt original des romans de Denise Mina réside dans l'évocation douloureuse de la situation de femmes qui ne peuvent ni envisager de retourner une Irlande lointaine 
qu'elles ne connaissent pas et sous peine de briser leurs liens familiaux, ni ne pas subir un climat délétère dans le monde du travail. Mais David Clark conclut que des changements profonds se dessinent ainsi qu'il le précise à propos des romans de Denise Mina qui «[...] show a Scotland in the process of change, in which the industrial strength of the nation is becoming a thing of the past. The newspaper industry is undergoing technological changes which will affect its entire ethos, as the exigencies of selling "product" replace the ethical code of previous decades » (p. 149)

Le dernier article du recueil écrit par Paddy McAnena (University of the West of Scotland) à la fois apporte une forme de conclusion aux propos qui le précèdent tout en formulant une interrogation sous le titre «Is the Scottish Work Ethic Fit for the 21st Century?». Évoquant pour mémoire la position prédominante de l'industrie lourde en Écosse au $\mathrm{XIX}^{\mathrm{e}}$ siècle et dans la première moitié $\mathrm{du} \mathrm{XX}^{\mathrm{e}}$, suivie par son effondrement dans la seconde moitié, l'auteur explique que l'activité économique en est aujourd'hui venue à reposer surtout sur le secteur tertiaire. Le domaine de la finance subit de plein fouet le choc de la crise des années 20072008. Se fondant sur les thèses de G. Hofstede, Paddy McAnena écrit : «Using Hofstede's socio-cultural dimensions, it is possible to argue that it is unreasonable to expect a workforce that has been on the wrong end of the employer-employee power distance divide for so long to be able to provide entrepeneurs quickly as large organisations disappear without replacement. Added to this disavantage, it is also possible to identify fear of failure (uncertainty avoidance) to the reasons why the Scottish work ethic does not result in higher entrepeneurial activity. » (p. 157)

Tous les articles de cette revue sont accompagnés d'excellentes bibliographies particulièrement précieuses pour le chercheur. Leur haut niveau, la rigueur des analyses, et leur présentation diachronique en font un outil de grande valeur pour travailler sur ce thème majeur qu'est l'éthique du travail en Écosse dans le passé et de nos jours.

\section{Pierre Morère}

Univ. Grenoble Alpes

Christophe Roncato, Kenneth White, une xuvre-monde, avec une préface de Régis Poulet, Presses universitaires de Rennes, publié avec le soutien le GEMRA, université Stendhal-Grenoble 3, 2014, 247 pages, 19 euros. 